\title{
Pancreatic ductal adenocarcinomas with cystic features: neither rare nor uniform
}

\author{
Markus Kosmahl, Ursula Pauser, Martin Anlauf and Günter Klöppel \\ Department of Pathology, University of Kiel, Germany
}

\begin{abstract}
Cystic tumors of the pancreas are uncommon but important because of their diverse pathology and biology. Their wide spectrum also includes cystic variants of otherwise solid tumors, such as cystic endocrine tumors, cystic acinar cell carcinomas and ductal adenocarcinomas with cystic changes. In this study, we screened pancreatic ductal adenocarcinomas and their variants for macrocystic changes and determined the nature of the cysts (neoplastic vs non-neoplastic). Of 483 tumors $38(8 \%)$ had cystic features. The largest group consisted of 24 pancreatic ductal adenocarcinomas showing a large-gland pattern with small cysts whose diameter varied between 0.5 and $1.8 \mathrm{~cm}$. The epithelial lining of these cysts was generally positive for CEA (83\%) and/or MUC1 $(71 \%)$ and MUC5AC (74\%). p53 was positive in $57 \%$ of the cases. The second group of cystic tumors $(8 / 483)$ showed degenerative cystic cavities with diameters ranging between 1 and $6 \mathrm{~cm}$. This group consisted of poorly differentiated pancreatic ductal adenocarcinomas, undifferentiated carcinomas with or without osteoclast-like giant cells and one adenosquamous carcinoma. In the third group of cystic tumors there were four pancreatic ductal adenocarcinomas containing tumor-related retention cysts. Their epithelial cells were positive for MUC5AC, but negative for CEA, MUC1 and p53. The fourth group consisted of two pancreatic ductal adenocarcinomas showing closely attached pseudocysts caused by tumor-associated pancreatitis. The results indicate that a considerable number of pancreatic ductal adenocarcinomas and their variants display cystic features and must therefore be considered in the differential diagnosis of cystic neoplasms of the pancreas. Moreover, not all of the cystic structures we observed were neoplastic in nature. They may also represent nonneoplastic changes, such as retention cysts and inflammatory pseudocysts.
\end{abstract}

Modern Pathology (2005) 18, 1157-1164. doi:10.1038/modpathol.3800446; published online 27 May 2005

Keywords: pancreas; cystic tumors; ductal adenocarcinomas

The majority of pancreatic neoplasms have a solid growth pattern and are ductal adenocarcinomas. ${ }^{1}$ Cystic neoplasms are rare, but they form an important group because of their special pathology and biology. The most common cystic tumors are intraductal papillary-mucinous neoplasms, serous cystic neoplasms, mucinous cystic neoplasms and solid pseudopapillary neoplasms., ${ }^{2,3}$ Rare cystic neoplasms include acinar cell cystadenocarcinomas, ${ }^{4}$ acinar cell cystadenomas, ${ }^{5}$ cystic endocrine tumors $^{6}$ and cystic mesenchymal tumors. ${ }^{7}$ The differential diagnosis of pancreatic cystic neoplasms, however, also includes pancreatic ductal adenocarcinomas with cystic changes. Most of the cystic pancreatic ductal adenocarcinomas reported in the literature were poorly differentiated tumors showing pseudocystic changes. ${ }^{8-10}$ Other pancreatic ductal adenocarcinomas with a predominantly

Correspondence: Dr M Kosmahl, MD, Department of Pathology, University of Kiel, Michaelisstr. 11, Kiel 24105, Germany.

E-mail: mkosmahl@path.uni-kiel.de

Received 8 February 2005; revised 11 April 2005; accepted 12 April 2005; published online 27 May 2005 microcystic appearance caused by marked ectasia of neoplastic glands were termed 'large-duct type' invasive carcinoma. ${ }^{11,12}$ Since pancreatic ductal adenocarcinomas with cystic features have not yet been systematically studied, we screened a series of 483 tumors consisting of pancreatic ductal adenocarcinomas and their variants, such as adenosquamous carcinomas and undifferentiated carcinomas with and without osteoclast-like giant cells, for macrocystic changes and analyzed the cause and nature of these structures.

\section{Materials and methods}

The pancreatic tumors reviewed in this series were identified in the surgical pathology files and the consultation files of the Department of Pathology of the University of Kiel. Clinical information was obtained from the patients' records and in some cases from the clinicians in charge. The review spanned the period from 1971 to 2003. All neoplasms had been resected. Carcinomas with an associated pre-existing cystic neoplasm (mucinous 
cystic neoplasm, serous cystic neoplasm, intraductal papillary-mucinous neoplasm) were excluded from the study.

The tumor tissue was fixed in $10 \%$ formaldehyde solution and embedded in paraffin. Deparaffinized sections were stained with hematoxylin and eosin and periodic acid-Schiff. From each case one or two tissue blocks were analyzed immunohistochemically using the primary antisera listed in Table 1. Immunohistochemical analysis was carried out on serial sections using the avidin-biotin-peroxidase technique (ABC ELITE, Vector Laboratories, Burlingame, CA, USA). Endogenous phosphatase activity was blocked with $1 \mathrm{mM}$ levamisole in the substrate solution. The slides were either directly immunostained or first pretreated by incubation with protease or boiling in citrate-buffered saline for $3.5 \mathrm{~min}$.

\section{Results}

A total of 483 pancreatic ductal adenocarcinomas and variants were screened for macrocystic changes.
Among these, 38 pancreatic ductal adenocarcinomas with cystic features were found. They included $24(63 \%)$ pancreatic ductal adenocarcinomas with large-gland features, eight (21\%) pancreatic ductal adenocarcinomas with intratumoral degenerative cystic changes, four (11\%) pancreatic ductal adenocarcinomas with retention cysts and two (5\%) pancreatic ductal adenocarcinomas with attached pseudocysts. They occurred more often in women (22 cases) than in men (16 cases). The ages of the patients ranged from 43 to 78 years with a mean of 62 years. Table 2 summarized the clinicopathological data and Table 3 the immunohistochemical results on all cystic pancreatic ductal adenocarcinomas.

\section{Pancreatic Ductal Adenocarcinomas with Large-Gland Features}

Most of these tumors contained multiple (more than five) cystic structures (with diameters ranging from 0.4 to $1.8 \mathrm{~cm}$ ) (Figure 1a). Only a few tumors had

Table 1 Antibodies used to immunostain cystic pancreatic ductal adenocarcinoma

\begin{tabular}{|c|c|c|c|c|c|}
\hline $\begin{array}{l}\text { Antigen/ } \\
\text { antibody }\end{array}$ & Host sp. & Source & $\begin{array}{l}\text { Working } \\
\text { dilution }\end{array}$ & $\begin{array}{l}\text { Antigen } \\
\text { retrieval }\end{array}$ & Control tissue \\
\hline CEA & Mouse & DAKO, Glostrup, Denmark & $1: 100$ & Boiling & Colorectal carcinoma \\
\hline P53 & Mouse & Oncogene, San Diego, CA, USA & $1: 20$ & Boiling & Breast carcinoma \\
\hline KiS5 & Mouse & Department of Hematopathology, University of Kiel & $1: 100$ & Boiling & Tonsil \\
\hline MUC1 & Mouse & Novo-Castra, Newcastle, UK & $1: 100$ & Boiling & Mammary \\
\hline MUC2 & Mouse & Novo-Castra & $1: 100$ & Boiling & Duodenum \\
\hline MUC5AC & Mouse & Chemicon Int., Temecula, CA, USA & 1.500 & Boiling & Gall bladder, stomach \\
\hline MUC6 & Mouse & Novo-Castra & $1: 100$ & Boiling & Stomach \\
\hline
\end{tabular}

Table 2 Pancreatic ductal adenocarcinomas (PDACs) with cystic features, clinicopathological data

\begin{tabular}{|c|c|c|c|c|c|c|}
\hline Tumor type & $\mathrm{N}$ & Age (years) & Sex & $\begin{array}{l}\text { Tumor size, cm } \\
\text { (range, mean) }\end{array}$ & $\begin{array}{l}\text { Cyst size, cm } \\
\text { (range) }\end{array}$ & Localization of tumor \\
\hline PDAC, large-gland type & 24 & $58(47-77)$ & $9 \mathrm{~m} / 15 \mathrm{f}$ & 2-15 (4.5) & $0.3-1.8$ & 12 head, 6 body, 3 tail, 3 pancreas \\
\hline PDAC, degenerative cystic type & 8 & $59(43-78)$ & $4 \mathrm{~m} / 4 \mathrm{f}$ & $1.3-19(9)$ & $1-6$ & 1 head, 1 body, 2 tail, 4 pancreas \\
\hline PDAC with retention cyst & 4 & $65(59-69)$ & $1 \mathrm{~m} / 3 \mathrm{f}$ & $1.3-2.5(2)$ & $0.5-1.5$ & 4 head \\
\hline PDAC with attached pseudocyst & 2 & $60(54,65)$ & $2 \mathrm{~m}$ & $4-4.5(4.3)$ & 1 & 1 head/1 body/tail \\
\hline
\end{tabular}

Table 3 Frequency of marker expression (\%) in cystic pancreatic ductal adenocarcinomas (PDACs)

\begin{tabular}{|c|c|c|c|c|c|c|c|}
\hline & MUC1 & MUC2 & MUC5 & MUC6 & $C E A$ & $P 53^{\mathrm{a}}$ & $K i S 5^{\mathrm{b}}$ \\
\hline PDAC, large-gland type & $17 / 24(71)$ & $2 / 24(1)$ & $17 / 23(74)$ & $12 / 23(52)$ & 19/23 (83) & $13 / 23(57)$ & $1-60(20)$ \\
\hline PDAC, degenerative cystic type & $6 / 8(75)$ & $0 / 8(0)$ & $2 / 8(25)$ & $4 / 8(50)$ & $3 / 8(38)$ & $6 / 8(75)$ & $2-80(37)$ \\
\hline \multicolumn{8}{|l|}{$P D A C$ with retention cyst } \\
\hline Tumor & $4 / 4(100)$ & $0 / 4(0)$ & $3 / 3(100)$ & $1 / 3(33)$ & $3 / 3(100)$ & $3 / 4(75)$ & $10-30(23)$ \\
\hline Retention cyst epithelium & $0 / 3(0)$ & $0 / 3(0)$ & $3 / 3(100)$ & $2 / 3(66)$ & 1/3 (33) & $0 / 4(0)$ & $0-2(1)$ \\
\hline PDAC with attached pseudocyst & $1 / 2(50 \%)$ & $0 / 2(0)$ & $2 / 2(100)$ & $1 / 2(50)$ & $1 / 2(50)$ & $0 / 2(0)$ & $10(10)$ \\
\hline Total (PDAC) & 28/38 (74) & $2 / 38(1)$ & $24 / 36(67)$ & $18 / 36(50)$ & $27 / 36(75)$ & $22 / 37(60)$ & $1-80(23)$ \\
\hline
\end{tabular}

\footnotetext{
ap53, cutoff level 5\%.
}

${ }^{\mathrm{b}}$ KiS5: range (mean). 

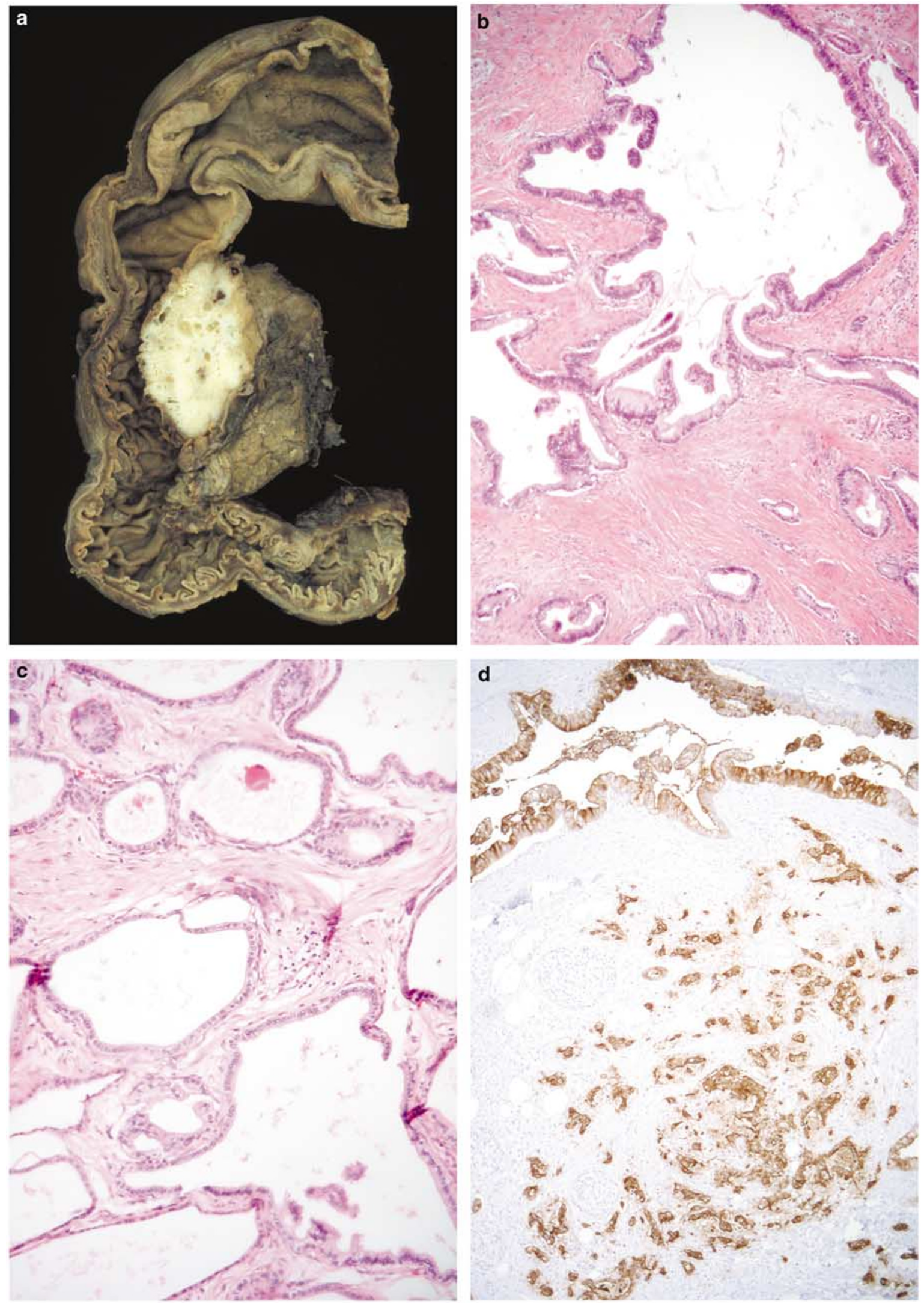

Figure 1 Pancreatic ductal adenocarcinoma, large-gland type. (a) Whipple resection specimen showing a tumor with multiple small intratumoral cysts. (b) Neoplastic microcystic structures embedded in desmoplastic stroma. (c) Numerous small cysts forming a honeycomb pattern. (d) The cells of the microcystic structures and the smaller tumor glands stain intensively for CEA. 
fewer than five small or large cysts (maximum $7 \mathrm{~cm}$ ). The cysts were usually scattered within the tumors, but were occasionally located at the tumor periphery. Histologically, they were lined by atypical cuboidal to flat epithelial cells occasionally forming papillary projections. The cystic structures were embedded in paucicellular desmoplastic stroma that bore no resemblance to ovarian-like stroma. It contained small irregular neoplastic duct-like glands (Figure 1b) as well as a few remaining acini, ducts and islets. According to the differentiation of the small duct-like neoplastic glands, 13 pancreatic ductal adenocarcinomas were classified as well differentiated, seven as moderately differentiated and two as poorly differentiated. ${ }^{12}$ In addition to cystic structures one ductal adenocarcinoma showed dilated mucin-filled glands and two others had a large squamous component. The neoplastic cysts were lined by atypical squamous cells. The latter two tumors, which represented adenosquamous variants of pancreatic ductal adenocarcinomas, contained the largest cysts observed in this tumor group. Two pancreatic ductal adenocarcinomas were characterized by numerous small cysts forming a honeycomb-like pattern (Figure 1c). Some of these cysts were lined by cuboidal cells with clear cytoplasm like those seen in serous cystic neoplasms, ${ }^{12,13}$ but most cysts had the mucus-producing epithelium that is typical of pancreatic ductal adenocarcinomas, in some cases with papillary projections.

Immunohistochemically, the pancreatic ductal adenocarcinomas with large-gland features stained for CEA (83\%) (Figure 1d) and/or MUC1 (71\%) in both their cystic structures and their noncystic duct-like glands. These components usually stained for MUC5AC (74\%) and MUC6 (52\%). Only two pancreatic ductal adenocarcinomas stained faintly for MUC2. Of the two adenosquamous carcinomas only one was weakly positive for MUC1 and CEA in its glandular component. Non-neoplastic duct epithelium was often positive for MUC5AC and MUC6. Nuclear p53 reactivity was seen in the neoplastic epithelium of $13 / 23$ tumors. The proliferation rate of the tumor cells varied between 1 and $60 \%$ (mean $20 \%$ ).

In all, 12 pancreatic ductal adenocarcinomas were localized in the head of the pancreas, six in the body and three in the tail. The tumors had a mean size of $4.5 \mathrm{~cm}$ (range $2-15 \mathrm{~cm}$ ). They occurred in nine men and 15 women, with a mean age of 58 years (range 47-77 years). Clinical data were available for 8 patients. Three patients had a history of pancreatitis; one patient presented with unspecific abdominal pain and in two patients the tumor was detected incidentally during a radiological examination. One patient had a preoperative diagnosis of a pseudocyst because a large cyst $(7 \mathrm{~cm}$ in diameter) had been detected in the tail of the pancreas. Another patient had symptoms of intestinal obstruction, and imaging revealed cystic structures in the region of the pancreatic tail involving the colon wall.

\section{Pancreatic Ductal Adenocarcinomas with Degenerative Cystic Changes}

Each of these eight tumors contained one large central pseudocystic cavity that usually contained some necrotic and hemorrhagic tissue (Figure 2a). The size of the cysts varied from 1 to $6 \mathrm{~cm}$ (mean $3 \mathrm{~cm}$ ). The wall of the pseudocysts was composed of tumor cells that showed hemorrhagic necrosis at the inner surface of the cyst (Figure 2b).

Two of the tumors in this group were moderately differentiated pancreatic ductal adenocarcinomas, one of them showing a transition to a pleomorphic large cell carcinoma and one a poorly differentiated adenosquamous carcinoma. Most of the cases (five) were undifferentiated carcinomas; three of them were classified as undifferentiated carcinomas with osteoclast-like giant cells (including the case reported by Oehler et $a l^{14}$ ), one as an undifferentiated carcinoma with large pleomorphic cells and one as a medullary carcinoma.

Immunohistochemically, the pancreatic ductal adenocarcinomas with degenerative cystic changes stained for MUC1 (75\%), MUC5AC (25\%) and MUC6 $(50 \%)$. None of the tumors stained for MUC2. CEA stained $38 \%$ of the tumors. The proliferation rate of the tumor cells ranged from 2 to $80 \%$ with a mean of $37 \%$. p53 was positive in $75 \%$ of all cases.

The tumors were localized in the head (one case), body (one case) and tail (two cases) of the pancreas. The medullary carcinoma (with a maximum diameter of $19 \mathrm{~cm}$ ) was attached to the pancreas and extended to the stomach and duodenum. In four cases the tumors were localized in the pancreas but the precise localization was unknown. The tumor size varied from 1.3 to $19 \mathrm{~cm}$ (mean size $9 \mathrm{~cm}$ ). Pancreatic ductal adenocarcinomas with degenerative cystic changes were observed in four women and four men (mean age 59 years, range 43-78 years). Clinical data were available in six cases. Three patients presented with abdominal pain. One patient was operated on for a suspected pseudocyst; one had a history of pancreatitis and one presented with weight loss and a periumbilical mass.

\section{Pancreatic Ductal Adenocarcinomas with Retention Cysts}

Four pancreatic ductal adenocarcinomas, two of them moderately differentiated and two poorly differentiated, showed unilocular cysts outside the tumor. The size of the cysts varied from 0.5 to $1.5 \mathrm{~cm}$. The cysts were lined by flat ductal epithelium without atypia (Figure 3). Sometimes the epithelium was denuded. Two cases showed mucinous metaplasia (PanIN-1A). Focal pericystic inflammation and fibrosis were seen.

Immunohistochemically, the tumor tissue reacted strongly with MUC1 (100\%), MUC5AC (100\%), CEA $(100 \%)$ and p53 (75\%). MUC6 was positive in 33\% 


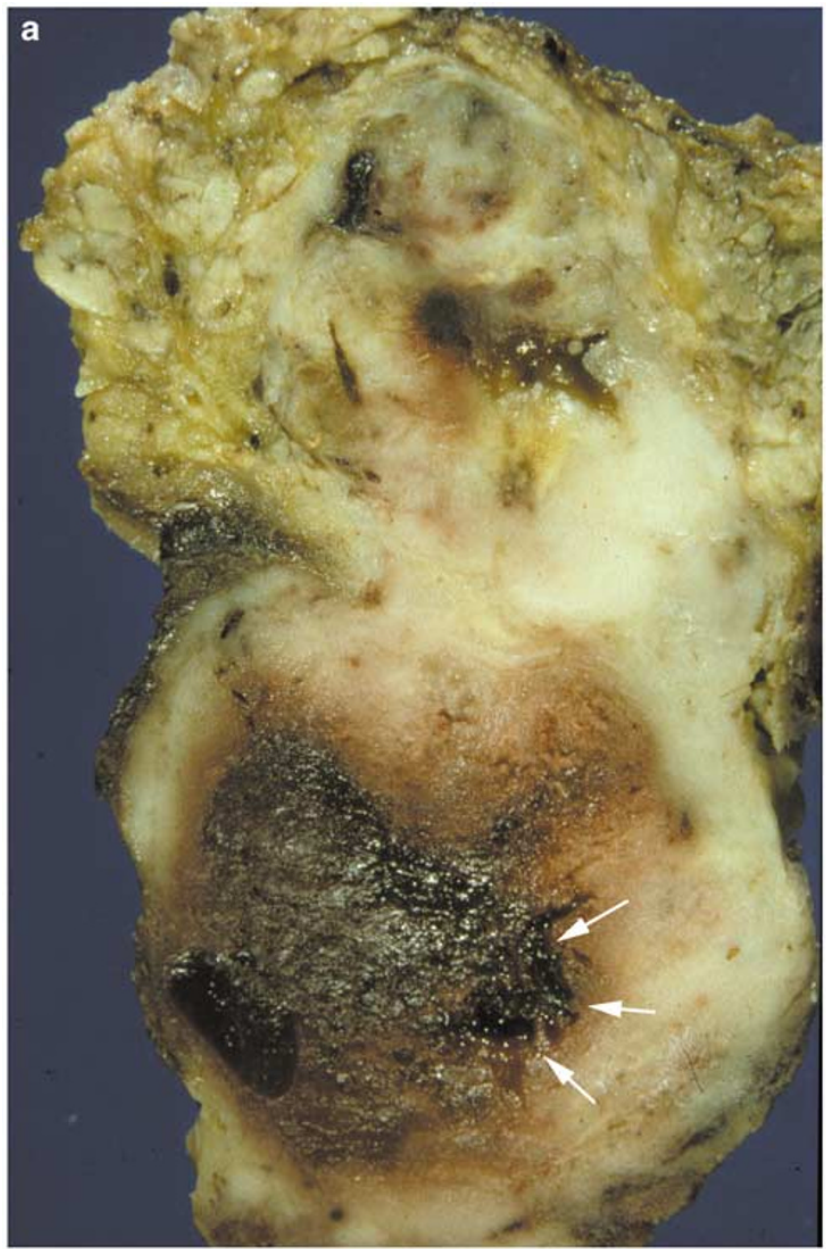

b

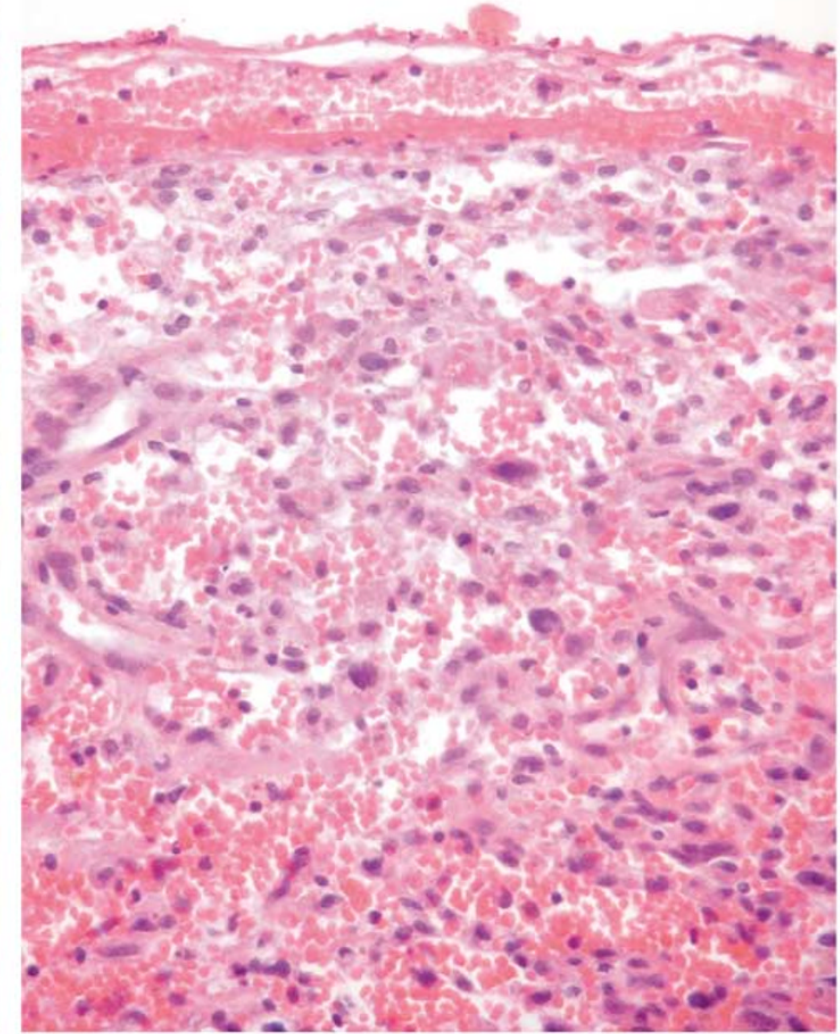

Figure 2 Undifferentiated carcinoma of the pancreas. (a) Whipple resection specimen showing central pseudocystic cavity with hemorrhage (arrows). (b) Cyst wall composed of pleomorphic tumor cells that show hemorrhagic necrosis at the inner surface of the cyst.

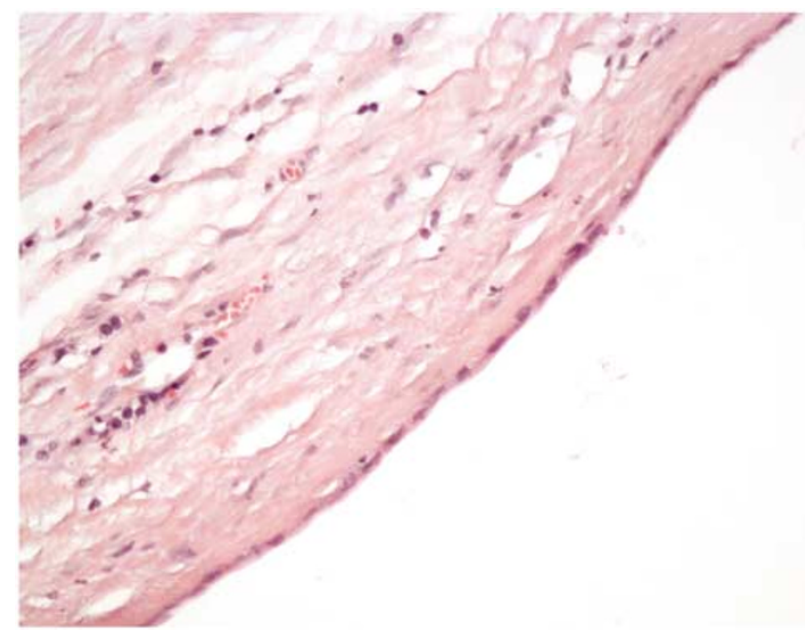

Figure 3 Wall of a retention cyst in a pancreatic ductal adenocarcinoma showing mucinous epithelium supported by hyalinized stroma. No atypia.

of the cases and MUC2 was negative. The proliferation rate ranged from 10 to $30 \%$ with a mean of $23 \%$.
The epithelium of the retention cysts stained strongly for MUC5AC (100\%). MUC6 was positive in $66 \%$ of the cases. No immunoreactivity was seen for MUC1, MUC2 and p53. In one case, focal positivity for CEA was seen. The proliferation rate was very low (mean $1 \%$ ).

Pancreatic ductal adenocarcinomas with retention cysts were observed in three women and one man (mean age 65 years, range 59-69 years). No information on the clinical presentation was available. All tumors were localized in the head of the pancreas. The tumor size varied from 1.3 to $2.5 \mathrm{~cm}$ (mean size $2 \mathrm{~cm}$ ).

\section{Pancreatic Ductal Adenocarcinomas with Attached Pseudocysts}

Two pancreatic ductal adenocarcinomas, both moderately differentiated, were found to have pseudocysts. Both cysts were unilocular, localized outside the tumor and $1 \mathrm{~cm}$ in size. The cysts were lined by necrotic tissue with hemorrhagic material and turbid fluid. No epithelial lining was detected. The cysts were surrounded by inflammatory tissue. 
Immunohistochemically, both tumors reacted strongly with MUC5AC. MUC1, MUC6 and CEA stained one of the cases, the other case was negative (the block was 25 years old). Both tumors were negative for MUC2 and p53. The proliferation rate was $10 \%$.

Both pancreatic ductal adenocarcinomas affected men (54 and 65 years). Both patients were known to have chronic pancreatitis. One tumor was localized in the head of the pancreas and one in the body/tail region. The tumors were 4 and $4.5 \mathrm{~cm}$ in size.

\section{Discussion}

Most pancreatic ductal adenocarcinomas and their variants are solid tumors. ${ }^{1,12}$ In the cases in which cystic transformation was reported, the tumors were found to have either pseudocystic changes $^{8-10,14-20}$ or simple cysts. ${ }^{21}$

In our large series of pancreatic ductal adenocarcinomas, including their variants such as adenosquamous carcinoma and undifferentiated carcinoma, $8 \%$ of the tumors showed cystic features. This implies that cystic changes are not a rare occurrence in pancreatic ductal adenocarcinomas and their variants and therefore must be included in the differential diagnosis of cystic neoplasms and lesions of the pancreas. In a recent study on cystic neoplasms and lesions of the pancreas we were able to show that pancreatic ductal adenocarcinomas with cystic features accounted for $7 \%$ of all cases. ${ }^{3}$ Since similar figures have also been reported by other authors, ${ }^{16,17,21}$ pancreatic ductal adenocarcinomas with cystic features are among those cystic neoplasms that have to be considered, when a tumorous cystic change of the pancreas is detected by imaging procedures.

As described in the literature cited above, and confirmed by us, the cystic changes found in association with pancreatic ductal adenocarcinomas differed in nature and pathogenesis. In almost twothirds of the tumors in our study the cystic structures represented a neoplastic component of the pancreatic ductal adenocarcinomas. This was demonstrated by the expression of the markers $\mathrm{CEA}^{22}$ and/or MUC1 ${ }^{23}$ in the epithelial cells lining the cystic structures. Another marker that labeled about $60 \%$ of the cases was p53. ${ }^{24-26}$ The expression of these markers was particularly helpful in cases in which the epithelium of the cystic structures was extremely flattened and therefore difficult to distinguish from the non-neoplastic epithelium of retention cysts. Cystic duct-like structures as an integral part of some pancreatic ductal adenocarcinomas were already described by Frantz ${ }^{27}$ and have been mentioned in the more recent literature. ${ }^{11,12,28}$ Usually, these pancreatic ductal adenocarcinomas were well differentiated, as was also observed in our series, and formed a small number of cavities with diameters in the range of $0.5-0.7 \mathrm{~cm}$ and only rarely exceeding $1 \mathrm{~cm}$. Although these changes are already grossly visible, they are difficult to detect with the currently available imaging techniques. The number of cavities in the individual pancreatic ductal adenocarcinomas varied from 5 to 15 . Pancreatic ductal adenocarcinomas with numerous small cystic structures showing a honeycomb pattern reminiscent of a serous adenoma are exceptions and were only seen twice in our series.

The second largest group in our series of pancreatic ductal adenocarcinomas and their variants with cystic features consisted of eight neoplasms that developed their cystic changes because of extensive central tumor necrosis. Most of these tumors (five cases) had a high proliferation rate and were classified as undifferentiated pleomorphic carcinomas (with or without osteoclast-like cells) or as poorly differentiated pancreatic ductal adenocarcinomas. Since these neoplasms were large (mean diameter $9 \mathrm{~cm}$ ) and showed one big pseudocystic cavity that often contained hemorrhagic debris and had an irregular outline, they could be easily distinguished grossly from pancreatic ductal adenocarcinomas with large-gland components. These features may also help to identify the pancreatic ductal adenocarcinomas with degenerative cystic changes when using imaging techniques.

The third group of tumors was small and encompassed only four pancreatic ductal adenocarcinomas characterized by single small $(0.5-1.5 \mathrm{~cm})$ retention cysts, all of them localized at the tumor periphery. They were distinguished from largegland-type pancreatic ductal adenocarcinomas by their negativity for the markers CEA, MUC1 and p53. All of them stained for MUC5AC (which is known to be expressed in most pancreatic carcinomas with columnar epithelium, but is also found in non-neoplastic proliferations of the duct epithelium $^{29}$ ) and some also for MUC6. The peripheral localization of these cysts suggested that they had been caused by duct obstruction due to the tumor.

The fourth and last group consisted of two pancreatic ductal adenocarcinomas, each with a pseudocyst attached to the tumor. Both patients presented with symptoms of pancreatitis, suggesting that the pseudocyst developed as a result of a necrotizing inflammatory process in conjunction with the neoplasm. The cause of this tumorassociated pancreatitis is not known, but from a number of reports it is obvious that acute pancreatitis in association with a pancreatic neoplasm is not rare and occurs in 5\% of pancreatitis patients. ${ }^{30-34}$ It is assumed that duct obstruction by the tumor is an initiating factor in this type of pancreatitis.

The differential diagnosis of pancreatic ductal adenocarcinomas and their variants with cystic features focuses on the first and fourth groups, that is, the pancreatic ductal adenocarcinomas that have a cystic neoplastic component and the pancreatic ductal adenocarcinomas with retention cysts, because the neoplasms in the other groups are easily 
distinguished on the basis of their macroscopic and microscopic features from the most important and common cystic neoplasms of the pancreas, such as intraductal papillary-mucinous neoplasms, mucinous cystic neoplasms and serous cystic neoplasms. ${ }^{3}$ Intraductal papillary-mucinous neoplasms, in contrast to 'large-gland type' pancreatic ductal adenocarcinomas, are localized in the main pancreatic duct and have an intraductal papillary mucin-producing tumor component, which is often MUC1 negative and MUC2 positive. ${ }^{23,35}$ When invasive, the invasive component may assume the appearance of a usual ductal adenocarcinoma or a mucinous noncystic carcinoma. Intraductal papillary-mucinous neoplasms of branch-duct type can be distinguished from ductal adenocarcinomas by their frequent positivity for MUC5AC and negativity for MUC1. ${ }^{36}$

In summary, pancreatic ductal adenocarcinomas and their variants with cystic features are neither rare, nor do they form a uniform group. Instead, they occur in a considerable percentage of cases and show a spectrum of cystic changes ranging from neoplastic to non-neoplastic structures, each showing special features and its own pathogenesis.

\section{References}

1 Klöppel G, Hruban RH, Longnecker DS, et al. Ductal adenocarcinoma of the pancreas In: Hamilton SR, Aaltonen LA (eds). Pathology and Genetics of Tumours of the Digestive System. WHO Classification of Tumours. IARC Press: Lyon, 2000, pp 221-230.

2 Klöppel G, Kosmahl M. Cystic lesions and neoplasms of the pancreas. The features are becoming clearer. Pancreatology 2001;1:648-655.

3 Kosmahl M, Pauser U, Peters K, et al. Cystic neoplasms of the pancreas and tumor-like lesions with cystic features: a review of 418 cases and a classification proposal. Virchows Arch 2004;445:168-178.

4 Cantrell BB, Cubilla AL, Erlandson RA, et al. Acinar cell cystadenocarcinoma of human pancreas. Cancer 1981;47:410-416.

5 Zamboni G, Terris B, Scarpa A, et al. Acinar cell cystadenoma of the pancreas. A new entity? Am J Surg Pathol 2002;26:698-704.

6 Ligneau B, Lombard-Bohas C, Partensky C, et al. Cystic endocrine tumors of the pancreas: clinical, radiologic, and histopathologic features in 13 cases. Am J Surg Pathol 2001;25:760.

7 Lee JS, Kim HS, Jung JJ, et al. Ancient schwannoma of the pancreas mimicking a cystic tumor. Virchows Arch 2001;439:697-699.

8 Lee LY, Hsu HL, Chen HM, et al. Ductal adenocarcinoma of the pancreas with huge cystic degeneration: a lesion to be distinguished from pseudocyst and mucinous cystadenocarcinoma. Int J Surg Pathol 2003; 11:235-239.

9 Kimura W, Sata N, Nakayama $\mathrm{H}$, et al. Pancreatic carcinoma accompanied by pseudocyst: report of two cases. J Gastroenterol 1994;29:786-791.

10 Dennis JW, Aranha GV, Greenlee HB, et al. Carcinoma masquerading as a pancreatic pseudocyst on ultrasound. Am Surg 1984;50:334-339.
11 Adsay NV, Klimstra DS. Cystic forms of typically solid pancreatic tumors. Semin Diagn Pathol 2000;17:81-88.

12 Solcia E, Capella C, Klöppel G. Tumors of the Pancreas. AFIP Atlas of Tumor Pathology, third series, fascicle 20. Armed Forces Institute of Pathology: Washington, DC, 1997.

13 Kosmahl M, Wagner J, Peters K, et al. Serous cystic neoplasms of the pancreas: an immunohistochemical analysis revealing alpha-inhibin, neuron-specific enolase, and MUC6 as new markers. Am J Surg Pathol 2004;28:339-346.

14 Oehler U, Jurs M, Klöppel G, et al. Osteoclast-like giant cell tumour of the pancreas presenting as a pseudocyst-like lesion. Virchows Arch 1997;431:215-218.

15 Scott R, Jersky J, Hariparsad G. Case report: malignant giant cell tumour of the pancreas presenting as a large pancreatic cyst. Br J Radiol 1993;66:1055-1057.

16 Grieshop NA, Wiebke EA, Kratzer SS, et al. Cystic neoplasms of the pancreas. Am Surg 1994;60:509-514.

17 Martin I, Hammond P, Scott J, et al. Cystic tumours of the pancreas. Br J Surg 1998;85:1484-1486.

18 Nabae T, Yamaguchi K, Takahata S, et al. Adenosquamous carcinoma of the pancreas: report of two cases. Am J Gastroenterol 1998;93:1167-1170.

19 Kardon DE, Thompson LD, Przygodzki RM, et al. Adenosquamous carcinoma of the pancreas: a clinicopathologic series of 25 cases. Mod Pathol 2001;14: 443-451.

20 Colarian J, Fowler D, Schor J, et al. Squamous cell carcinoma of the pancreas with cystic degeneration. South Med J 2000;93:821-822.

21 Talamini MA, Pitt HA, Hruban RH, et al. Spectrum of cystic tumors of the pancreas. Am J Surg 1992;163: 117-123.

22 Bätge B, Bosslet K, Sedlacek HH, et al. Monoclonal antibodies against CEA-related components discriminate between pancreatic duct type carcinomas and nonneoplastic duct lesions as well as nonduct type neoplasias. Virchows Arch [A] Pathol Anat 1986;408: 361-374.

23 Lüttges J, Zamboni G, Longnecker D, et al. The immunohistochemical mucin expression pattern distinguishes different types of intraductal papillary mucinous neoplasms of the pancreas and determines their relationship to mucinous noncystic carcinoma and ductal adenocarcinoma. Am J Surg Pathol 2001;25: 942-948.

24 DiGiuseppe JA, Hruban RH, Goodman SN, et al. Overexpression of p53 protein in adenocarcinoma of the pancreas. Am J Clin Pathol 1994;101:684-688.

25 Boschman CR, Stryker S, Reddy JK, et al. Expression of p53 protein in precursor lesions and adenocarcinoma of human pancreas. Am J Pathol 1994;145:12911295.

26 Apple SK, Hecht JR, Lewin DN, et al. Immunohistochemical evaluation of K-ras, p53, and HER-2/neu expression in hyperplastic, dysplastic, and carcinomatous lesions of the pancreas: Evidence for multistep carcinogenesis. Hum Pathol 1999;30:123-129.

27 Frantz VK. Tumors of the Pancreas. AFIP Atlas of Tumor Pathology, first series, section 7, fascicles 27 and 28. Armed Forces Institute of Pathology: Washington, DC, 1959.

28 Motoo Y, Kawashima A, Watanabe H, et al. Undifferentiated (anaplastic) carcinoma of the pancreas showing sarcomatous change and neoplastic cyst formation. Int J Pancreatol 1997;21:243-248. 
29 Terada T, Ohta T, Sasaki M, et al. Expression of MUC apomucins in normal pancreas and pancreatic tumours. J Pathol 1996;180:160-165.

30 Lin A, Feller ER. Pancreatic carcinoma as a cause of unexplained pancreatitis: report of ten cases. Ann Intern Med 1990;113:166-167.

31 Greenberg RE, Bank S, Stark B. Adenocarcinoma of the pancreas producing pancreatitis and pancreatic abscess. Pancreas 1990;5:108-113.

32 Yachida S, Fukushima N, Nakanishi K, et al. Minute pancreatic adenocarcinoma presenting with stenosis of the main pancreatic duct. Pathol Int 2002;52: 607-611.

33 Imamura M, Asahi S, Yamauchi $\mathrm{H}$, et al. Minute pancreatic carcinoma with initial symptom of acute pancreatitis. J Hepatobiliary Pancreat Surg 2002;9: 632-636.

34 Zyromski NJ, Haidenberg J, Sarr MG. Necrotizing pancreatitis caused by pancreatic ductal adenocarcinoma. Pancreas 2001;22:431-432.

35 Longnecker DS, Adler G, Hruban RH, et al. Intraductal papillary-mucinous neoplasms of the pancreas In: Hamilton SR, Aaltonen LA (eds). Pathology and Genetics of Tumours of the Digestive System. WHO Classification of Tumours. IARC Press: Lyon, 2000, pp 237-240.

36 Adsay NV, Merati K, Basturk O, et al. Pathologically and biologically distinct types of epithelium in intraductal papillary mucinous neoplasms. Delineation of an 'intestinal' pathway of carcinogenesis in the pancreas. Am J Surg Pathol 2004;28:839-848. 